\title{
The astute resident and the IACUC
}

$\mathrm{T}$ here are so many issues with this scenario and the resident's actions, however, the key question is: should this issue be reported to OLAW and the USDA? Since "the PHS Policy and the Animal Welfare Regulations (AWRs) do not distinguish between animals owned by the institution and privately owned animals," we can assume that cats being used for research purposes are covered under both USDA and OLAW requirements. The original protocol uses privately owned veterinary patients, which normally require consent from the owners. In this case, no consent was obtained for the abandoned cat and it was used without the knowledge of the PI, Attending Veterinarian, or IACUC.

The Animal Welfare Act section $₫ 2158$ states that a licensed research facility should hold the cat for a period of at least 5 days in case the original owner returns. ${ }^{2}$

The cat was procured surreptitiously, brought into the animal facility, and housed inappropriately in a cat carrier, which provides significantly less space than the USDA regulations or The Guide state, and treated. ${ }^{3,4}$ The approved protocol did not include using atenolol in conjunction with the new drug. If we assume that the university's assurance covers all research animals regardless of funding, then the IACUC must report to OLAW any serious non-compliances. ${ }^{5}$

The institution should keep in mind that the OLAW and the USDA have a Memorandum of Understanding, agreeing to share information reported to them regarding animal welfare concerns; ${ }^{6}$ therefore, even if the institution reported the non-compliances to OLAW only, it can be presumed that the USDA would also be informed.

As for the resident, the IACUC's authority is to suspend an activity if warranted, not specific personnel; however, they bear responsibility for assuring that those working with animals are properly trained. The IACUC, during the special meeting, may choose to suspend the PI's protocol because of the non-compliances; this suspension would be reported to both OLAW and the USDA. Clearly the IACUC must discuss the actions of the resident, aside from the PI's protocol non-compliances. Were the resident's actions deliberate or just ignorant? Although the IACUC's authority does not cover HR issues, nor can they terminate someone, the IACUC can require that the resident not perform any more animal activities until the IACUC deems that the resident is proficient in animal welfare legislation and guidances, in the ethics of animal use, in the use of the three R's, and any other trainings the institution requires. Perhaps this resident should consider another line of research not involving animals.

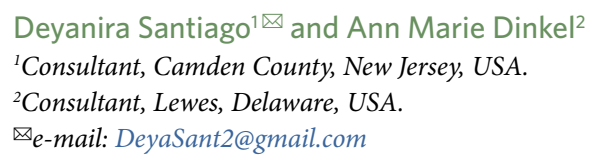

Published online: 20 August 2020 https://doi.org/10.1038/s41684-020-0620-3

\section{References}

1. Brown, P. \& Gipson, C. Lab Anim (NY) 38, 186 (2009).

2. Animal Welfare Act and Regulations 7 U.S.C. $₫ 2158$ (a)(1), (a) (2)(C)

3. Animal Welfare Act and Regulations 9 C.F.R. $\$ 3.6(\mathrm{~b})(1)(\mathrm{ii})(\mathrm{B})(\mathrm{C})$

4. Institute for Laboratory Animal Research. Guide for the Care and Use of Laboratory Animals. 8th ed. (National Academies Press, Washington D.C., 2011).

5. Public Health Service. PHS Policy on Humane Care Use of Laboratory Animals. IV.F.3 (U.S. Department of Health and Human Services, National Institutes of Health, Bethesda, MD 2015).

6. Office of Laboratory Animal Welfare. MOU Among USDA/ FDA/NIH (1 March 2006). http://grants.nih.gov/grants/olaw/ references/finalmou.htm

7. Animal Welfare Act and Regulations 9 C.F.R. $\$ 2.31(\mathrm{c})(8),(\mathrm{d})(7)$

\section{We've got 99 problems but prompt reporting ain't one of them}

T he scenario presented is riddled with problems, including violations of the Animal Welfare Act (AWA)

Regulations, animal welfare issues, IACUC protocol noncompliance, and possibly a deficiency in training.

The School of Veterinary Medicine at Great Eastern University has an OLAW Assurance and an USDA research registration; however, the clinical trial in question was supported by a non-federally funded grant. Assuming that the OLAW Assurance only covers PHS-funded studies, no report to OLAW is required. USDA only requires prompt reporting if a protocol is suspended or if there is nonadherence to a plan of correction for significant deficiencies identified during the IACUC semi-annual program review and facility inspection ${ }^{2}$. Although there are clear violations of the AWA, the scenario presented here does not meet the criteria for prompt reporting. In addition, there is no requirement to report the suspension of protocol staff to either agency.

While reporting to federal agencies is not required, the IACUC has plenty of work to do to investigate this incident, determine all aspects of noncompliance, and determine a correction plan and/or other mitigations to prevent this from happening again. Their most immediate concern should be to ensure that the cat is being properly cared for and that the resident is prevented from further work on this project until the incident is thoroughly investigated and discussed by the IACUC.

As an USDA registered research facility, Great Eastern University must comply with AWA regulations and standards, including provisions for lawful acquisition and compliance with animal health and husbandry standards. ${ }^{2}$ In this 